Juhani Hämäläinen, Ph.D (Physics), Chief Scientist at Finnish Defence Research Agency, juhani.hamalainen@mil.fi

Jari Sormunen, Lieutenant Colonel, G.S., Chief of Staff in South Finland Logistics Regiment, jari.sormunen@mil.fi

Jari Rantapelkonen, Professor, Department for Tactics and Operational Art, Finnish National Defence University, jari.rantapelkonen@mil.fi

Juha-Pekka Nikkarila, PhD (Physics), scientist at Finnish Defence Research Agency, juha-pekka.nikkarila@mil.fi

\title{
WARGAME AS A COMBINED METHOD OF QUALITATIVE AND QUANTITATIVE STUDIES
}

\section{Keywords}

Wargame, qualitative methods, quantitative methods, combined methods, experience.

\begin{abstract}
Wargames are important methods for military planning, education and research. Qualitative models have a long tradition in wargaming for improving and practising the military skills as well as enhancing the military planning based on the experiences and understanding of the experts. Quantitative models are included in simulations and technical studies. Traditionally, the relations between the qualitative and quantitative research have been described as opposites. We shall consider both the approaches, their challenges and possibilities, and their combination for producing more exhaustive wargame and for answering to the criticism of wargame as a mainly qualitative method.
\end{abstract}

Our main research question is: How to combine quantitative and qualitative approaches and methods to improve quality of results of military wargames? Our main objective is to clarify and illustrate how qualitative and quantitative methods can be combined through the wargame. We shall emphasize the importance of the model selection at the beginning as it limits and guides the form (not the content) of the obtained results. We shall illustrate a combined wargame within different steps and the modelling themes of the game. Our results and findings should be useful for improving and focusing wargames to answer given research questions. 


\section{Introduction to wargames}

Wargames provide a possibility to combine qualitative and quantitative research. The characteristics of the quantitative and the qualitative research orientations are many and not without difficulties to describe their ultimate definitions. In Table 1 we shall illustrate the key and simple characteristics.

Table 1. The relationship between the qualitative and quantitative research.

\begin{tabular}{|l|l|}
\hline QUALITATIVE RESEARCH & QUANTITATIVE RESEARCH \\
\hline Describing & Explaining \\
\hline Based on Human Sciences & Based on Natural Sciences \\
\hline Phenomenology-hermeneutics & Empirical-causal \\
\hline Soft research & Hard research \\
\hline Experienced based & Measurement based \\
\hline
\end{tabular}

From the ontological and epistemological viewpoint it is basically assumed that the research object really exists and it is possible to gain information about it both by observations and by intuition and that it is possible to process this information. In this research, the effort is to study the interrelationships of the existing things. The research process includes rationalization by utilizing induction, deduction and abduction as well.

The traditional military studies stress war and battle exercises as a method for acquiring new knowledge. Also military experiences and tacit knowledge of military experts (Huhtinen \& Rantapelkonen, 2001) are crucial for knowing more about the chaotic and complex battlespace.

Wargames are important methods for examining and evaluating military scenarios to study the general question "who would win the battle?" These can be used to compare alternative possibilities of tactics in different scenarios and they can support the military exercises or future operation plans. Wargames are usually carried through in experts' qualitative dialogue supported by a quantitative analysis. In the research wargames, the experiences of individual soldiers have a significant role for producing information. 
Modelling in wargames consists of studies from the technical level to the strategic level. It is challenging to construct solvable models which support military planning in a realistic manner. In principle, many models only emphasize or approximate the original problem, but are still valuable for improving the solution and for further development of the models. Military models combine the military understanding, the technical knowledge and many modelling techniques to provide a more comprehensive view of the given questions.

By mirroring the military hierarchy to the different models of the wargame, it can be stated that the qualitative analysis dominates at the upper levels in the hierarchy (strategic and operational studies) and quantitative analysis at the technical and tactical studies. In order to acquire useful information from the modelling, military and modelling experts should cooperate and discuss through the game by comparing the plans and the models and fitting them together. Usually, military experts provide and verify the initial states of the game which modellers support by suitable models. If suitable models are not available or they do not exist, model development is needed. The second iteration consists of studying the preliminary results and comparing them to the initial assumptions. This step can lead to more accurate modelling requirements or fixed initial conditions, again in cooperation. This will be repeated as long as the results are reliable enough for further analysis of the game.

Typical scenario consists of many details, which generate alternative wargames. This happens, because every parameter change generates a new alternative. This happens also with the timing of the tactical decisions. Naturally, all alternatives cannot be studied but the interest should be in the most probable and descriptive ones to consider them computationally e.g. by data farming (Horne \& Meyer, 2004).

We shall discuss here on the different kind of models and model selection in the pre-modelling phase. The selection procedure affects on what kind of results will be available for answering the research question and is therefore important for carrying out the research. 


\section{Hierarchy in wargaming}

Hierarchic structures appear naturally in military studies as it reflects the traditional structure of a military organization. However, hierarchy can be seen also in a computational tool or a modelling and a visualization technique for understanding the complex environment and the problems. We shall consider the hierarchic structures of the models and the parameters, which are not new but often encountered with computational structures. One widely applied method is the analytical hierarchy process (Vargas \& Whittaker, 1990), which might be useful for implementing computational models of the wargames.

\section{Scale of the models}

Modelling has its limitations due to the structure of the models, for instance a different scale of events requires a different scale (resolution) of the models. The scales are determined by the timing, the modelling resolution and the size of the scenario. Modelling limitation may follow from the available computational resources or from the internal structure of the model, because many models are constructed and validated for the special purposes. In practice, larger scale of the problem needs more complex model and more attention has to be paid to the implementation. One approach is to consider the complex problems with hierarchic structures by composing the models from the smaller sub-models (Kasputis \& Ng, 2000).

\section{Structure of parameters}

Parameters fix the initial values of the models and they have a significant role to describe given technical details, tactics, equipment and other relevant matters for the given problem. The importance of the parameters is highlighted as the output information of the models depends on them. In other words, inaccurate or incomplete information of the parameters provide unrealistic results even though the model would be correct. However, it is always important to know the accuracy and the constraints of the parameters and the models in order to ensure their correct usage range. The reliability, the validity and the history of the parameters should be documented i.e. why certain values are used and which this knowledge is based on.

The military models consist of different kinds of parameters depending on the details, the scale (resolution) and the purpose of the model. The resolution is 
usually higher, if the model describes technical details when compared with those dealing with tactics or other higher level entities. The hierarchy means that the results of more accurate models will be used as parameters in the higher level models (Hämäläinen, 2008), and the parameters and the models are categorized equally. This might confuse as the parameters and the variables of the models appear in a similar manner.

For emphasizing this idea, we consider an example related to the artillery studies. When one aims to examine the specific effects of different ammunition, accurate parameters of the initial velocity, information of the ammunition, fragmentation etc., are needed. These parameters are used to determine the effects of one grenade, which are usable parameters for modelling the artillery barrage. When this is conducted, study may continue to examine losses of a military unit under artillery fire. By combining this kind of combat events, one can continue to study bigger battle, where losses in different combats are examined at the higher level such that the final results can support to the analysis of a larger operation. In the artillery example, we shall call the losses of the unit to derived parameters and at the higher level to collected parameters, see Table 2.

Table 2: Example of a hierarchic structure of parameters.

\begin{tabular}{|l|l|l|l|}
\hline Parameter type & $\begin{array}{l}\text { Qualitative } \\
\text { example }\end{array}$ & $\begin{array}{l}\text { Quantitative } \\
\text { example }\end{array}$ & $\begin{array}{l}\text { Military } \\
\text { hierarchy }\end{array}$ \\
\hline physical parameter & $\begin{array}{l}\text { quality of } \\
\text { ammunition }\end{array}$ & $\begin{array}{l}\text { amount of } \\
\text { ammunition }\end{array}$ & Technical \\
\hline derived parameter & $\begin{array}{l}\text { description of a } \\
\text { strike }\end{array}$ & losses in a strike & Tactical \\
\hline collected parameter & $\begin{array}{l}\text { effects of strikes } \\
\text { to operations }\end{array}$ & $\begin{array}{l}\text { velocity of } \\
\text { operations }\end{array}$ & Operational \\
\hline
\end{tabular}

\section{Hierarchic models}

We described a hierarchic characterization of parameters. As the models need their parameterization, it is natural to characterize models equally. This means that the first level models tend to model the basic tasks with very accurate parameters like technical specifications. If the model is carefully validated and verified, the results will be accurate at this level. 
At the second level, modelling parameters can be the results of the first level calculations, but also additional parameters might be needed. Therefore, the representation of the results has more variations with wider perspectives than at the first level. At this level, smallest details are ignored since their effects will be lower than the error margin, which may appear difficult to determine. Also, these details may cause unclear features to the models and they may be difficult to verify. Many small effects may change the model's overall behaviour to an erroneous direction and further modelling becomes unreliable.

By continuing this procedure, we obtain a complete model, where the parameters are rougher and variations and errors of the results are more difficult to determine. This needs understanding of variety of applications, resolution and time scales (Kasputis and Ng, 2000). Tactics becomes more dominant and effects on the results and on the structures of the models. The complexity of the models tends to increase along with the hierarchic level. It concerns also the required time of the model's development, testing and validation.

\section{Qualitative wargames and models}

Qualitative wargames are based on an experts' dialogue in a two-sided refereed discussion on the battle. The advantage is that experts' knowledge is constantly available. On the other hand, it may lead to a subjective description if the game is carried out purely qualitatively. Qualitative models and methods deal with descriptive information and can be expressed in various forms, e.g. text, stillpicture, motion picture and voice (Perttula, 2006 and Varto, 2005).

Input information of the qualitative methods differs from the quantitative methods. It may be based on interviews, expert knowledge, experience, etc., and typically deals with observations or opinions of a professional soldier. Methods themselves organize information for instance by different grouping techniques (Miles and Huberman, 1994). A typical example of qualitative military modelling would be a tactical or an operational scenario constructed with expert professional knowledge. The scenario can be further studied quantitatively (combat modelling) or qualitatively (expert war game). Qualitative analysis cannot be repeated in details like quantitative one and the observations as well as the results depend on the experts. Therefore, systematic errors due to opinions are more probable and the results can be biased. Man-in-the-loop simulation may be used to combine the 
expert knowledge with the quantitative methods. Qualitative analysis might be illustrated in the following manner (Figure 1.) (Miles \&Hubermann, 1994).



Figure 1. An example of material-based qualitative analysis to be utilized in a wargame according to (Miles \& Hubermann, 1994).

\section{Quantitative wargames and models}

Quantitative wargame can be conducted with computer simulations (simulators), numerical analysis or other quantitative methods. More often, wargames include quantitative supporting parts. When there are needs for the computational support beforehand, during or afterwards of the game, preparations and plans of the analysis should cover also the computational requirements. Computational support needs software, specialized users and understanding what could or should be conducted. In short, computing should improve and validate the findings and improve the repeatability of the game.

High resolution models provide useful information for a wargamer in the specific situations like in the previous example of the artillery strikes. In the bigger context, high resolution models provide parameter information to the higher level models. The game needs a script written beforehand or guidelines to direct the game and the 
modelling. Analysis of the observations and the modelling results may show particular points of the game for improving it in further exercises.

Computation can be performed also afterwards. This approach can be used for a comparison of the results, for the validation of the models and the script of the game. This can also provide useful information for further studies.

Quantitative models deal with information in a numeric (measurable) form provided by the computational, analytic and statistical models and simulations. In the optimal case, the models are constructed and the parameters are based on the physical measurements. The language of the quantitative models is mathematics, which makes the obtained results repeatable within their error limits. In short, quantitative models provide suitable tools for the problems, which can be expressed in terms of mathematics.

In the military context, well known and widely applied examples of mathematics are the Lanchester Power Laws and the Lanchester Differential Equations introduced by Frederick W. Lanchester (Lanchester, 1916) and e.g. M. Kress (Kress, 2009). Typical applications of pure quantitative modelling can be also found from theoretical physics and scientific computing (Hausken, 2011). Quantitative analysis uses numeric input data to be considered according to logical/mathematical rules and provide numeric results.

For instance, in the combat modelling (Lappi, 2012), the initial state, the equipment and the troops information are given. However, during the modelling more comprehensive picture of the situation will develop and it might appropriate to iterate the modelling. The iterations need military experts to create and specify the plans and modellers to implement them into the computational form. In order to achieve success both should participate for the computational implementation such that the modelled picture corresponds to the questions of interest. At the beginning both should identify and accept the limitations of the computing as misunderstanding and mistakes in initial information may yield to the erroneous case studies. Ideally, when the modelling starts military experts can verify the computational state accurately enough. The qualitative analysis is often needed together with the quantitative analysis, e.g. in modelling tactical decisions. 
There exists much literature of the topic and a good introduction to quantitative models in military modelling is given by Metteri (Metteri, 2006) with its references.

\section{Method and model selection for military modelling}

Method and model selections are usually guided by the given research questions. As the military studies can be organized in a hierarchic manner, models often follow similar structure. At the technical level, quantitative models (analytic, numerical, simulations) are dominant and at higher levels qualitative models dominate, although there exist quantitative approaches also at the higher levels. However, many questions need both and combined approaches.

Possible methods for the given problem are selected in the pre-modelling phase which should be emphasized. The selection work will guide the overall problem solving through the process or a phase of it. Important subjects are the time scale where answers are needed, models' reliability, suitability, availability and using experience all with respect to the given question and the requirements. To improve the modelling and combining of the models, we suggest that the comprehensive model interpretation should be conducted from the very beginning.

We begin with the interpretation of the research question followed by the model selection and proceed to the comparison and the combination of different models. The combining needs mappings between the methods and in particular between the data. The purpose is to consider obtained information in a similar manner. A simple approach is to map input data of quantitative and qualitative models into equal form or to map quantitative data into qualitative or vice versa or to map the results into a common comparable form.

Next we move onto questions of how to select and combine the models. Qualitative and quantitative models differ significantly in their structure, data involved and in their results. Their using principles differ as well: qualitative analyses can be executed faster but their uncertainties may be larger. Often the quantitative analysis requires more time and its costs are higher (Metteri, $2006 \mathrm{p} .15$ ). The methods are also called hard (quantitative) and soft (qualitative) methods (Flood and Carson, 1993). 
In the complex problems both methods are often needed. Qualitative analysis is nearly always present at the beginning. Quantitative analysis is conducted e.g. when evaluating the impact of own or the opposite side. Quantitative analysis may be conducted e.g. by a simple numerical evaluation or via simulations. Examples of combined models can be found e.g. in the man-in-the-loop (Sandis, KESI) simulations, where the simulation software is based on quantitative models and operator adds information to the simulation. Also hardware-in-the-loop models exist in the analysis of the combination of different models. In the hardware-in-theloop analysis a part of the defence system is considered through a real component and its measurement results are combined with numerical analysis/simulations. On the other hand, the man-in-the-loop models could be called as "modelled" hardware-in-the-loop models since usually there is a part of weapon system modelled numerically and combined with a decision maker's actions. The added information may come from different sources like operators experience (qualitative) or supporting simulations (quantitative) and used software also restricts the form information appropriate for simulation.

Nevertheless, it is evident that the combination of the qualitative and the quantitative analysis is needed in the evaluation of the defence systems, at all levels from technical to strategic.

The role of the automated quantitative analysis (simulations, to be more specific) is to perform computations more efficiently than a human could do. It is important to remember that the responsibility of constructing and validating the models remains with human hands and the user has to ensure the applicability and the validity of the used models.

We shall now propose one process for method selection. The first step is to analyze a question, find out the time scale when answer is needed and focus the question into a form where it may be modelled (Figure 2) 


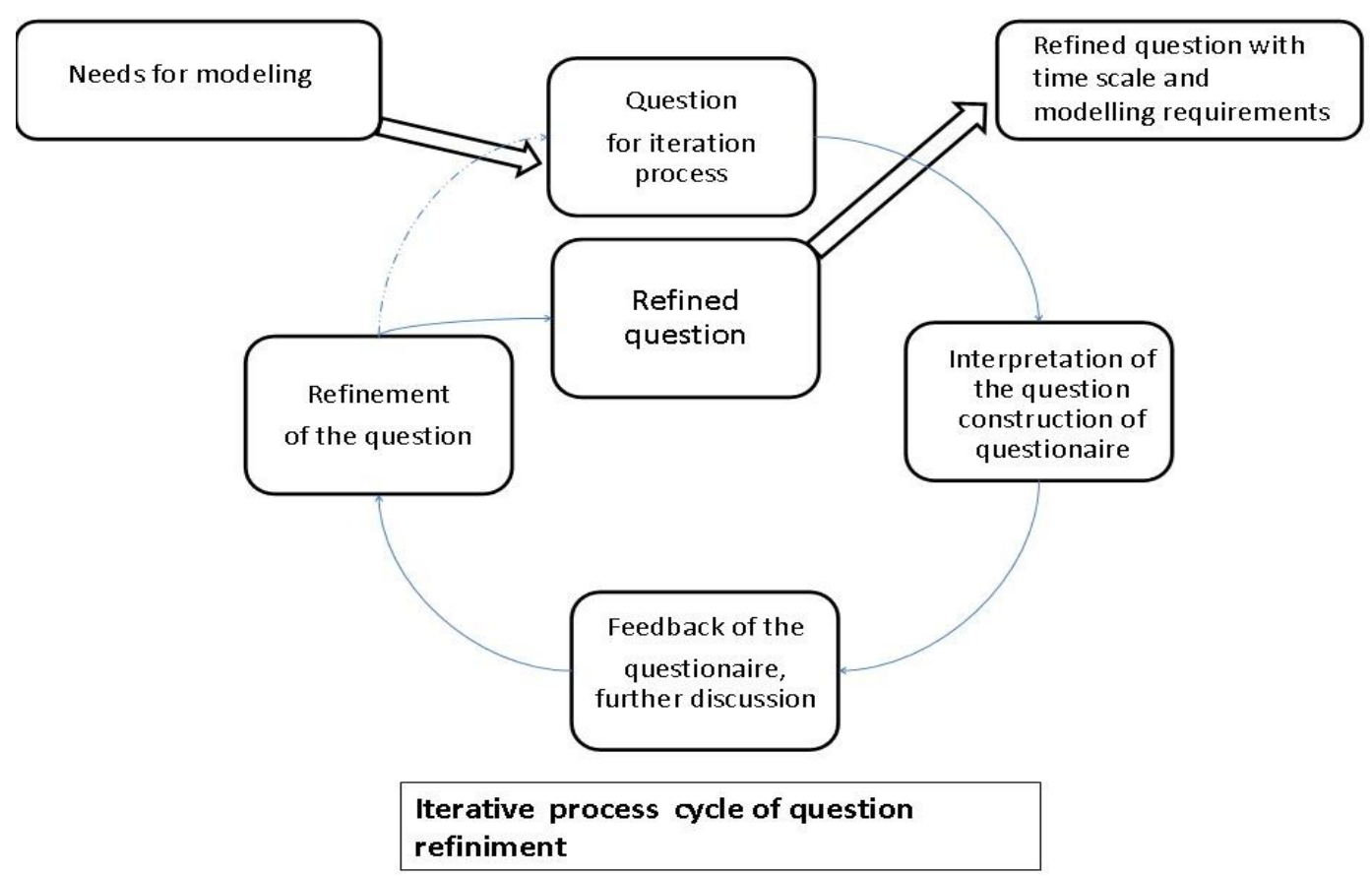

Figure 2. Description of iterative process in problem refinement from the modelling needs to refined problem statement (Hämäläinen, 2011).

The second step is to select appropriate models for the refined question according to the following proposed list.

- Determine the military level of the question, i.e., strategic, operational, tactical or technical and the time scale where the answer is needed.

- Identify suitable model candidates, their availability and existing using practise and experience as well as available modelling resources.

- Select the most appropriate models and also consider status of the models, i.e. their reliability for instance how the models are validated and verified.

If the question and the requirements can be expressed or translated into simulation models, model selection can be conducted by comparing software candidates with user requirements and research questions, see also (Kasputis \& Ng, 2000).

One may proceed systematically (quantitatively) if needed by valuating the model suitability, model availability, experiences of models' usage etc. and calculating 
comparison points for each model candidate. However, the described systematic would support the model selection but it is not a necessary step. The reliability of the models should be considered by comparing their validation and verification as well as their user experiences. However, this step often leads to need for model development where the model will be strictly focused to the given problem.

Models are useless without any input data and the diversity of the applied models leads to the diversity of the input data. This yields questions "how to compare different data" or "is the comparison sensible at all?" and "how reliable the given data is?" This should be taken into account in the error and sensitivity analysis of the results. If the comparison cannot be carried through, errors due to different input data should be documented carefully.

The problem of commensurate input data, if not solved, goes through models leading to the result representation with several attributes. This is still better way to express results than forcing them into one quantity, which may be misleading in the interpretation phase. The final interpretation should be conducted together with the decision maker or the question should be re-focused.

\section{Combined interpretation of the wargame}

When both aspects are combined together and analyzed carefully, it is possible to use the benefits of the quantitative methods to strengthen weaknesses of qualitative analysis and vice versa. The combined analysis improves the interpretation, the reliability and the validity range of the results. It is possible to analyze the situation more carefully and to resolve the problem if the results are comparable but different. On the other hand, if both methods provide similar results independently, the results confirm each other. We shall illustrate the verification of the results with triangulation method, where different error sources are taken into account (Figure 3.), see also (Suoranta \& Eskola 1998) and (Lincoln \& Cuba 1989 p. 162 and 241 and Golafshani, 2003). 


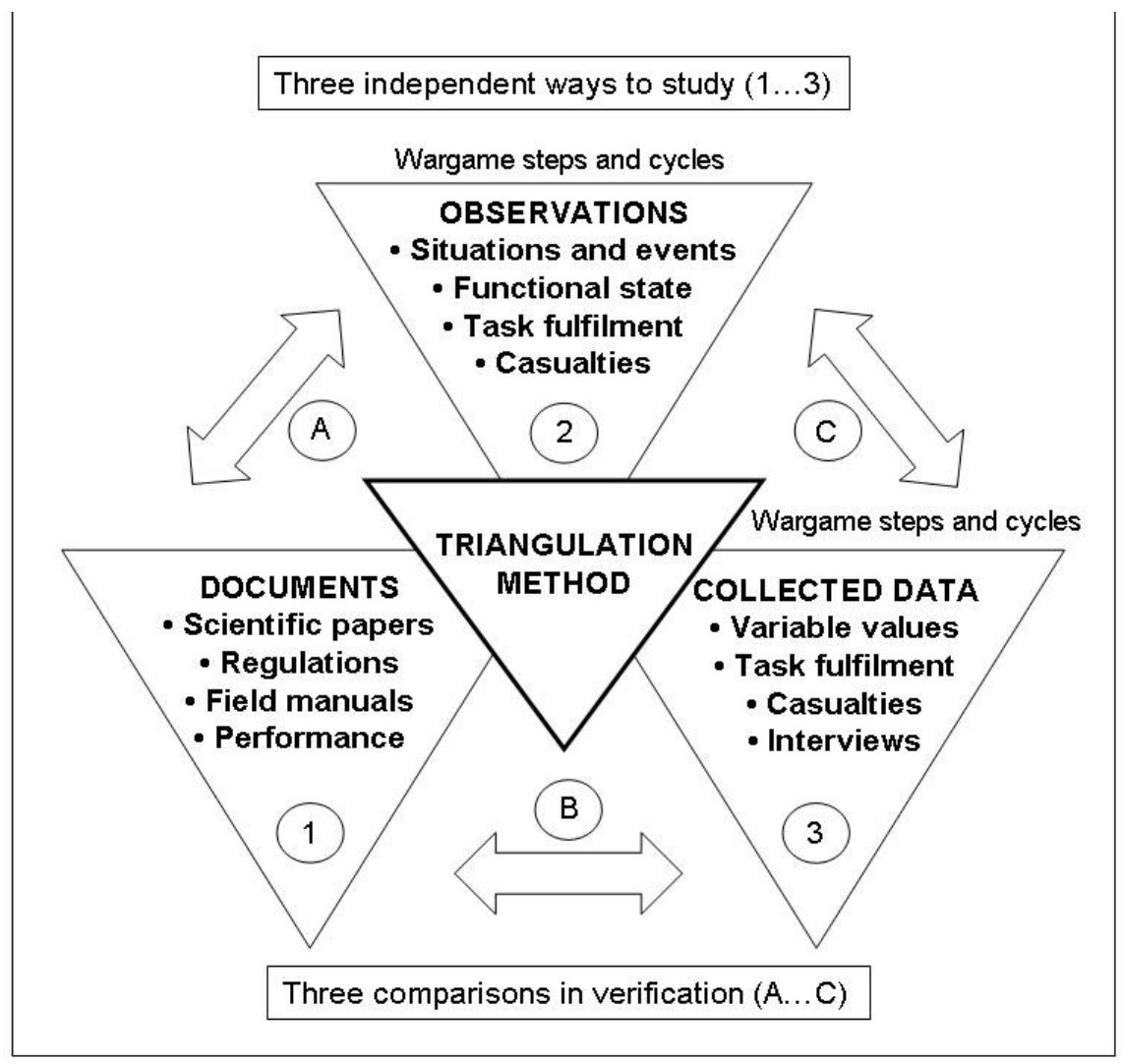

Figure 3. Example how to utilize the triangular method.

In the triangulation process, both the wargame players' and the researchers' observations about the situations and events, the units' functional states, task fulfilments and casualties are utilized as source data. In addition to this, one main part of the triangulation process in composed of the collected and measured data of the defined variables and the interviews of the wargame players. These data sources are utilized together with the previous information which consists of scientific papers, military regulations, field manuals and the performance data of the units.

As a wargame is a process, the methods selected at the beginning follow through the game and the errors/uncertainties cumulate during the game. Therefore, the importance of the model selection should be addressed. It will also help to manage the game more systematically and more transparently. When the combined analysis is conducted in different phases of the game, the risks of misleading results and unrealistic expectations decrease. 
Our purpose has been to examine the differences and similarities of qualitative and quantitative models and methods in a wargame. However, pragmatically oriented researchers refer to mixed methods containing elements of both approaches (Tashakkori \& Teddlie, 1998, p. ix-x and 3-18, Creswell, 2003, p.208-224 and Saunders et al, 2012, p. 126-153). Our main objective was to consider the methodological combination in a military wargame. We shall now present one proposition how the combination would be applied in the research wargame at the different states from the beginning to the results analysis. We observed how the research wargame should be focused pragmatically to answer the research questions within the given framework. At the beginning, the interpretation of the questions guides the model selection, which reflects to the form (not the content if valid models are applied) of the answers. During the game, different models and methods can be used, which should be considered in the analysis of the game. Our findings are collected in Figure 4.

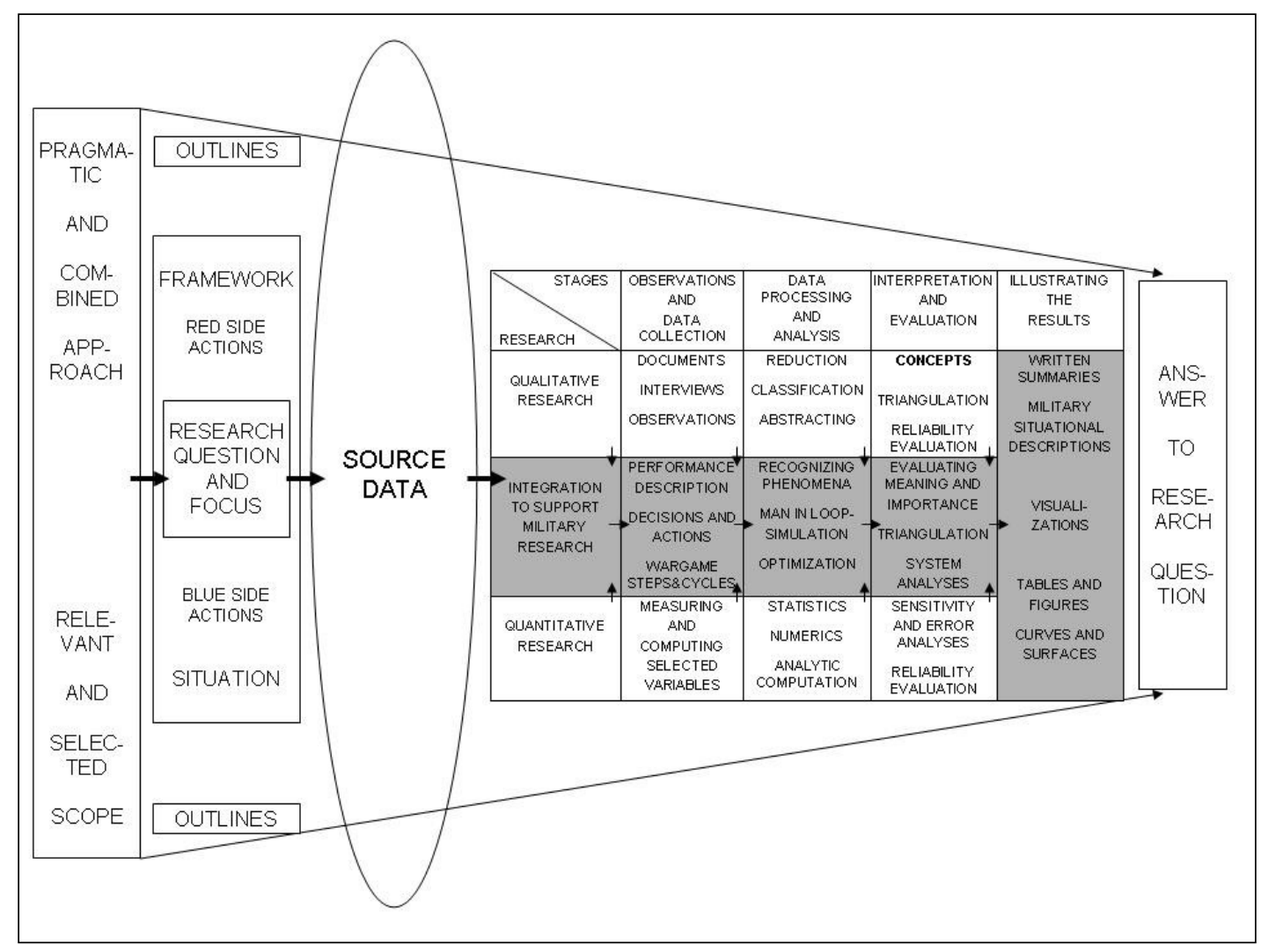

Figure 4. An illustration of the combination of different qualitative and quantitative methods in wargames' different phases. 
The figure illustrates some selected qualitative, quantitative and mixed methods and models applicable during the wargame. The method and model selection should begin at the early stages of the research wargame.

\section{An example}

Let us emphasize the illustrated method with a company against a company wargames. Let us assume that the selection procedure provides us the modelling software Sandis (Lappi, 2012), which makes this interpretation more concrete and the steps further can be utilized with Sandis. The guidelines in a Sandis wargame will be provided in this example and more detailed example can be found in (Bruun et al, 2010), where an ambush of a convoy is analyzed with respect to different tactical decisions.

Let us consider the case where one tactics is fixed and another tactics is varied. The question is, which tactics provides the best outcome for the varied side. The source data contains information of the equipments, troops and rough scenario which will be played. It also covers parameter information needed and previous documentations of related games.

At the first stage appropriate models should be selected and the base scenario should be described carefully. The next step includes a model implementation such that it can be varied and a construction of alternative tactics which will be played in separate scenarios. Based on this, a wargame can be conducted in a set of man-inloop simulations where military professionals provide the tactical maneuvers and modelling specialists implement the variations.

The outcomes of the games should be carefully documented all in a similar manner for a comparison analysis. The sensitivity of the results with respect to the used parameters or performed actions in tactical alternatives can be studied e.g. by data farming (Horne\&Mayer,2004). This should result on a set of outcomes (for both sides) in each alternative tactical scenario. Finally, the results appear e.g. as a set of strength distributions of played troops, the amounts of used ammunitions or simply the expectations of the losses of both sides in different tactical scenarios. These results should be analyzed again together in order to answer the question which tactics was the most successful for varied side. 


\section{Conclusions and discussion}

Qualitative and quantitative methods are used in wargames separately and together. Qualitative methods are well suited for research question development and focusing the research. They are proper methods for constructing the initial tactical or operational military situation and scenario and producing focused observations during the game steps and cycles, based on professional military experience.

Quantitative methods use and produce information in a measurable form, which is easier to compare and analyze systematically. On the other hand, the exactness of quantitative studies might mislead if the limitations and the range of the validity of the methods are not taken into account. However, the information collected in wargames has a large diversity which might confuse. Therefore, systematic means to collect and filter the obtained data are needed for the basis of the analyses and results interpretation.

The computational modelling in wargames often needs both quantitative and qualitative initial information but simultaneously provides possibilities to deepen and widen the game. In particular, computing can be used to study the details of the game within the models' restrictions. Typically, smaller events are easier to model than large scenarios and the resolution of the model depends on the size of the modelled situation.

We have also discussed on the method and model selection for military analysis and proposed to conduct a pre-study for the selection. The topic is worth studying in order to prevent erroneous results or research. Also, the pre-study ensures that e.g. the following aspects or questions are taken into account: "do we know what we are doing?", "why we are going to do so?", and "where the obtained results may be applied?". We encourage for a careful model comparison before the selection in order to meet and understand the questions and the associated requirements clearly. If this procedure is conducted in co-operation of the military specialists and modellers, both should benefit as it clarifies the questions as well as the modelling possibilities and limitations, i.e., models' applicability.

Our purpose was to examine and identify the advantages of combining qualitative and quantitative studies in the context of a research wargame. We introduced the combined interpretation of methods for clarifying the relations between qualitative 
and quantitative methods. It seems obvious that both aspects should be utilized together in a research wargame as these methods support and contribute to each other (Bruun et al, 2010 and Huhtinen \& Rantapelkonen, 2001). These findings should be helpful in constructing forthcoming wargames in a systematic manner and also for improving the quality of the research e.g. in documentation, transparency and repeatability. In the forthcoming studies, it would be interesting to apply also hardware-in-the-loop methods in the wargames.

\section{References}

Bruun R. S., Hämäläinen J. S., Lappi E. I. And Lesnowicz Jr E. J. (2010). Data Farming with SANDIS Software Applied to Mortar Vehicle Support for Convoys, Scythe, Proceedings of the International Data Farming Workshop 21: 18-21, Lisbon.

Creswell J. W. (2003). Research Design - Qualitative, Quantitative and Mixed Methods Approaches, 2003.

Eskola, J. and Suoranta, J. Johdatus laadulliseen tutkimukseen, Vastapaino, 1998.

Flood R. L. and Carson. E. R. (1993). Dealing with Complexity $2^{\text {nd }}$ ed., Plenum Press.

Golafshani N. (2003). Understanding Reliability and Validity in Qualitative Research, The Qualitative Report 8(4): 597-607 (http://www.nova.edu/ssss/QR/QR8-4/golafshani.pdf.).

Hausken K. and Zhuang J. (2011). Governments' and Terrorists- Defence and Attack in a T-period Game, Decision Analysis, 8(1): 46-70.

Horne G.E. and Meyer T.E. (2004), Data farming: discovering surprise, Proceedings of the 2004 Winter Simulation Conference, Washington.

Huhtinen A. \& Rantapelkonen J. (2001). Taistelut, kokemus ja tieto. Näkemys sotatieteellisestä viestitaktiikasta. Viestikoulu, Loimaa.

Hämäläinen J. S. (2008). Hierarchic parameter structures for military operational analysis, Proceedings of $2^{\text {nd }}$ Nordic Military Analysis Symposium, Stockholm.

Hämäläinen J. S. (2011). Focus group: data farming in support of strategic operational analysis, Scythe: Proceedings of International Data Farming Workshop 23, : 29-32, Riihimäki.

Kasputis S., and Ng H.C. (2000), Composable simulations, Proceedings of the 2000 Winter Simulation Conference, Washington:. 1577-1584.

Kress M. and Szechtman R. (2009). Why Defeating Insurgencies Is Hard: The Effect of Intelligence in Counterinsurgency Operations -A Best-Case Scenario, Operations Research 57(3): 578-585 
Lanchester F. W., Aircraft in Warfare: the Dawn of the Fourth Arm (1916)

Lappi E. (2012). Computational methods for tactical simulations, Doctor of Military Science thesis, National Defence University, Department of Tactics and Operations Art, Publications.

Lincoln, Y.S. and Guba, E.G. Fourth Generation Evaluation, SAGE Publications, 1989.

Metteri J. (2006). Kvantitatiiviset tutkimusmenetelmät operaatiotaidon ja taktiikan tutkimuksessa, Maanpuolustuskorkeakoulu, Helsinki.

Miles M. B. and Huberman M. A. (1994). Qualitative Data Analysis, $2^{\text {nd }}$ ed., 1994.

Perttula J. (2006). Kokemus ja kokemuksen tutkimus: Fenomenologisen erityistieteen tieteenteoria. p. 115-163 in Perttula, Juha - Latomaa, Timo (toim.) Kokemuksen tutkimus: Merkitys - tulkinta - ymmärtäminen. Helsinki: Dialogia Oy.

Saunders M., Lewis P. and Thornhill A. (2012), Research Methods for Business Student, $6^{\text {th }}$.

Tashakkori A. and Teddlie C. (1998). Mixed methodology - Combining qualitative and quantitative approaches, Applied Social Method,. 46.

Vargas, L.G. and Whittaker, R.W. (Eds) (1990). Decision making by the analytic hierarchy process: Theory and applications. European Journal of Operational Research, 48(1): 1-173.

Varto, Juha (2005). Laadullisen tutkimuksen metodologia. Elan Vital. 